\title{
LAURENT DEMANZE, Un nouvel âge de l'enquête. Portraits de l'écrivain contemporain en enquêteur
}

\section{Stefano Genetti}

\section{(2) OpenEdition}

\section{Journals}

\section{Edizione digitale}

URL: https://journals.openedition.org/studifrancesi/45478

DOI: 10.4000/studifrancesi.45478

ISSN: 2421-5856

\section{Editore}

Rosenberg \& Sellier

\section{Edizione cartacea}

Data di pubblicazione: 1 août 2021

Paginazione: 403-404

ISSN: 0039-2944

\section{Notizia bibliografica digitale}

Stefano Genetti, "Laurent demanze, Un nouvel âge de l'enquête. Portraits de l'écrivain contemporain en enquêteur», Studi Francesi [Online], 194 (LXV | II) | 2021, online dal 01 septembre 2021, consultato il 15 octobre 2022. URL: http://journals.openedition.org/studifrancesi/45478 ; DOI: https://doi.org/10.4000/ studifrancesi. 45478

Questo documento è stato generato automaticamente il 15 octobre 2022

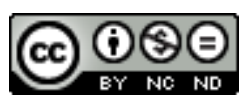

Creative Commons - Attribuzione - Non commerciale - Non opere derivate 4.0 Internazionale - CC BYNC-ND 4.0

https://creativecommons.org/licenses/by-nc-nd/4.0/ 


\title{
LAURENT DEMANZE, Un nouvel âge de l'enquête. Portraits de l'écrivain contemporain en enquêteur
}

\author{
Stefano Genetti
}

\section{NOTIZIA}

LAURENT DEMANZE, Un nouvel âge de l'enquête. Portraits de l'écrivain contemporain en enquêteur, Paris, Corti, 2019, «Les essais», 292 pp.

1 Di argomento affine al libro di F. Pouillaude di cui sopra, poiché «l'enquête littéraire est à inscrire dans l'importance actuelle des écritures factuelles» (p. 12), è la stimolante indagine di L. Demanze sulle implicazioni poetiche e politiche dell'indagine retrospettiva o ancorata al presente, individuale o partecipativa - in quanto dominante dell'odierna prosa narrativa. Tra i temi affrontati, ritroviamo l'esigenza documentaria e il paradigma indiziario, le problematiche legate alla trascrizione delle interviste, la dialettica di soggettività e oggettività; tra i testi trattati, ritroviamo l'equidistanza fra immedesimazione e istanza giudicante nell'Adversaire di Carrère, i montaggi di testimonianze di Hatzfeld, le anti-giornalistiche derive periurbane di Rolin. Volutamente meno teorico e sistematico è invece l'impianto esposto nell'Introduzione: è una mappatura critica che l'A. fornisce, attenta ai reticoli che intessono fra loro i vari percorsi creativi presi in esame e che si riflettono nel circolare dei riferimenti tra $i$ capitoli intitolati non a categorie rigidamente delimitate, bensì ad atteggiamenti ricorrenti: «S'étonner», «Explorer», «Collecter», «Restituer», «Poursuivre». Sensibile all'interazione delle «écritures de terrain» (p. 25) con le «interventions situées» (p. 113) che portano le arti visive fuori dagli spazi deputati, così come col laboratorio di scrittura la letteratura sconfina dal libro sul territorio, l'approccio adottato si rivela attento ai «braconnages disciplinaires» (p. 167), all'osmosi tra sapere antropologico, storiografico e sociologico che il proliferare delle inchieste comporta, determinando 
un'estensione - consapevole, autoriflessiva e rigorosamente documentata - e un decentramento discorsivo e istituzionale dei vari campi, a iniziare da quello letterario. A monte del reportage e della non-fiction, si situa il modello investigativo ereditato dall'Ottocento, impostosi e via via diluitosi con l'instaurarsi della democrazia e della cultura di massa, connesso allo sviluppo delle scienze sociali, alla diffusione del noir poliziesco e del racconto ermeneutico in senso lato, che la Recherche proustiana prolunga e sovverte (pp. 43-50). Tipico dell'«âge de l'enquête» di cui Zola constatava l'avvento (p. 11) e teso a oggettivare e a classificare, a decriptare e a razionalizzare, esso rappresenta piuttosto un contro-modello conoscitivo e narrativo per le numerose e diversificate pratiche repertoriate in questo volume, opportunamente corredato di Indice dei nomi.

2 Se il corpus include anche la critique policière di Pierre Bayard o la contre-enquête Meursault di Kamel Daoud, palinsesto postcoloniale basato sull'Étranger di Camus e oscillante tra omaggio e rivalsa, la maggior parte dei testi analizzati risulta riconducibile a tre raggruppamenti. In primo luogo, le inchieste a carattere biografico, dove vita propria e altrui (Vie et mort de Paul Gény, prozio di Philippe Artières), vissuto intimo e storia collettiva ( $L a$ cache di Christophe Boltanski) non di rado si intersecano e dove il rifiuto della finzione non esclude il ricorso all'immaginazione come deposito di rappresentazioni. Dalle contro-inchieste di Didier Blonde ai collage d'archivio di Artières, sono frammentari, lacunosi e talora ipotetici «exercices d'empathie» (p. 23). Se in Histoire des grands-parents que je n'ai pas eus di Ivan Jablonka, autore di L'histoire est une littérature contemporaine, alla vicenda ricostruita si affianca la vicenda della sua ricostruzione, la rom inseguita in Anthropologie da Éric Chauvier, cui si deve il saggio Anthropologie de l'ordinaire, si colloca nel fantomatico filone che da Nadja di Breton arriva a Dora Bruder di Modiano. In secondo luogo, le esplorazioni geografiche: viaggi di prossimità (Jean-Christophe Bailly, Le dépaysement) ed erranze periferiche che dello spazio urbano perlustrano gli interstizi e i margini, inducendo «une dénaturalisation du regard, un déconditionnement des habitudes, un réagencement de la syntaxe politique» (p. 99). Nell'ambito del programma Écrivains de la Seine-Saint-Denis Hélène Gaudy si concentra sulla vita quotidiana in un luogo della memoria quale Drancy. In Un livre blanc, Philippe Vasset colma le lacune, i blancs, delle carte topografiche della regione parigina: ben presto, la ricerca del merveilleux che presiede a questa geografia alternativa cede il posto alla denuncia delle sacche di invisibilità sociale, finché anche quest'ultima sfocia nell'impossibilità di rappresentare, inscritta sotto forma di ekphrasis delle fotografie scattate dall'autore durante le sue spedizioni. Vettori di questa «littérature déplacée» (p. 88) che cartografa non-luoghi di fatto abitati, animati - la stazione ferroviaria in Montparnasse-monde di Martine Sonnet o gli spazi inaccessibili al pubblico in Paris Gare du Nord di Joy Sorman - sono i mezzi di trasporto, dalla linea Parigi-Nancy in Paysage fer di François Bon a Les Passagers du Roissy-Express di François Maspero e Anaïk Frantz. In terzo luogo, le raccolte polifoniche, frutto di résidences e ateliers d'écriture spesso rivelatori di tensioni socio-economiche: tra gli operai che patiscono la disindustrializzazione (Jean-Paul Goux, F. Bon) o tra gli abitanti di una cité destinata alla demolizione (Maryline Desbiolles, C'est pourtant pas la guerre), tra le donne che hanno subito violenza (Nicole Malinconi, Hôpital silence) o in carcere (Prison di F. Bon, Maison d'arrêt Paris-La Santé di Olivia Rosenthal), si tratta di abbandonare «la position de surplomb de la littérature engagée» (p. 27) e di inventare «une politique de la forme qui intègre dans la composition le regard d'autrui, pour ne pas prendre la parole à la place d'autrui, mais construire un espace de langage avec eux» (p. 178). 
3 Nel loro insieme, i Portraits de l'écrivain contemporain en enquêteur allineati da L. Demanze gli permettono di disegnare i contorni etici ed estetici di una poetica, plurale ma identificabile, che fa dell'inchiesta «un marqueur de reconnaissance littéraire, d'affiliation générique et de positionnement épistémologique» (p.14). È qui che il richiamo alla tradizione ottocentesca risulta funzionale a mettere in luce, per contrasto, i tratti distintivi del nouvel âge de l'enquête in cui viviamo. Alla superiorità del detective solitario che risolve un enigma subentra l'immersione interattiva e solidale dello scrittore nel mondo: travalicando i confini del suo cabinet, egli corre il rischio del dilettantismo, dell'esperienza sul campo e dell'incontro. All'«ambition de normalisation $\mathrm{du}$ corps social» (p. 27) sottesa al paradigma inquisitoriale in quanto strumento di controllo subentra l'attenzione alle trame e agli strappi del tessuto sociale. All'imperativo dell'oggettività e all'ostentazione di un sapere accertato ed esauriente subentrano gli interrogativi sulle condizioni che regolano la percezione e la restituzione del reale, l'evidenziazione dei margini di opacità, di indicibilità. In conclusione (l'«Épilogue» si intitola «Suspendre» e si pone sotto l'egida di Perec esploratore dell'infra-ordinario), è alla sua sospensione, vale a dire al sopravvento che il movimento della ricerca prende sui suoi stessi esiti, che l'inchiesta letteraria contemporanea tende, sospesa com'è tra la ricognizione e l'incognita, tra «l'erratisme de l'essai» e «l'épiphanie poétique» (p. 266). 